\title{
3D Analysis of Large Volumes Through Automated Serial Sectioning
}

\author{
David J. Rowenhorst ${ }^{1}$, Lily Nguyen ${ }^{1,2}$, and Richard W. Fonda ${ }^{1}$ \\ 1. U.S. Naval Research Laboratory, Code 6350, Washington, DC, USA \\ 2. NRC Research Postdoctoral Associateship, Washington, DC, USA
}

The typical method for characterizing structural materials on the micron scale has primarily been performed on polished 2D cross-sections using a variety of microscopy techniques including optical microscopy (typically combined with an etchant), electron microscopy, and increasingly with analytical electron beam techniques such as Electron Dispersive Spectroscopy (EDS) and Electron Backscattered Diffraction (EBSD). Examining 2D cross-sections with stereological techniques can provide average values for a structure, but can not reveal some of the most critical aspects such as true sizes and shapes, full distributions, spatial correlations, and frequency of objects in the structure.

Recent advances, primarily due to increases in computational resources and automation, have lead to a suite of 3D techniques for the analysis of materials across length scales that range from nanometers to millimeters. However, many of these techniques can not effectively collect large volumes while maintaining a high resolution, thus making statistical evaluations difficult. One exception to this is the technique of serial-sectioning, wherein a consecutive set of closely spaced parallel cross-sections are analyzed with 2D microscopy and are stacked and aligned to provide a three-dimensional representation of the microstructure. While it is assumed that most of the effort in performing this type of analysis is associated with the collection of the serial-sections, the true time and effort is usually due to the segmentation of the hundreds to thousands of images.

In this work, we show that by incorporating a fully automated polishing station - which performs the material removal between sections - with a fully automated scanning electron microscope equipped with EBSD, much of this effort can be greatly reduced in polycrystalline materials, which allows for much larger datasets to be analyzed in reasonable timeframes. By being able to collect EBSD maps on every section, nearly all of the image processing becomes automated at the cost of much longer collection times. We will discuss how time is budgeted between removal rates, imaging time, and required resolution, and how post processing the data can further reduce collection times.

Here we will specifically examine the 3D structure of an Additively Manufactured (AM) 316L stainless steel. The microstructure formed from the rapid solidification that occurs during the powder-bed fusion process is highly complex, as shown in Figure 1 which shows one of the 300 Backscattered Electron (BSE) images and EBSD maps that were collected. The total volume collected was $900 \times 900 \times 400$ $\mu \mathrm{m}^{3}$ with a section thickness of $1.44 \mu \mathrm{m}$. From these images, we can see that while the BSE images are dominated by contrast from small changes in the crystallographic orientation, the EBSD images provide clear delineation of the grain boundary interfaces. However, the BSE images clearly shows the location of the pores produced during the printing process, thus the two datasets together provide a more complete description of the microstructure. Figure 2 shows a 3D reconstruction of the pore structure in the material, with the color of each region representing the spherical equivalent radius. From this reconstruction, we can see that the highest number of pores are relatively small $(<10 \mu \mathrm{m})$ and are typically spherical. However, there are a number of larger pores within the volume that show highly irregular shapes. We believe that these are locations in between laser passes of the printing laser that do not experience full remelting from 
subsequent layers. Future work will concentrate on removing geometric distortions from the EBSD data stack so that the two datasets can be fused and co-analyzed. [1]

\section{References:}

[1] This work was funded by the Naval Research Laboratory under the auspices of the Office of Naval Research and from the Structural Metallics program of ONR.
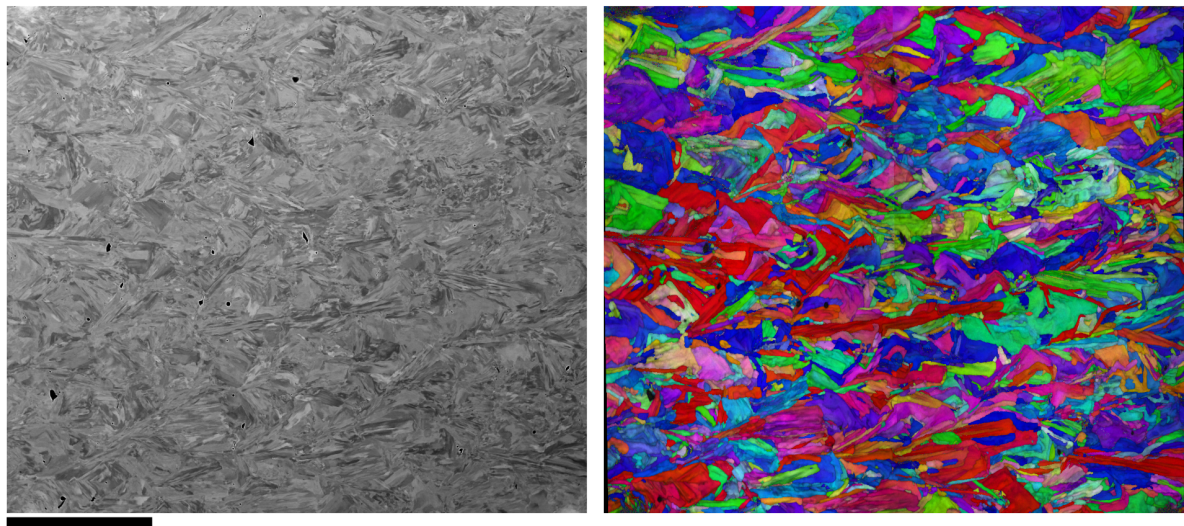

$300 \mu \mathrm{m}$

Figure 1. Co-registered BSE and EBSD map of AM processed 316L. The EBSD map reveals the complex grain morphology, while the BSE images provide high-resolution measurement of the pores from the printing process.

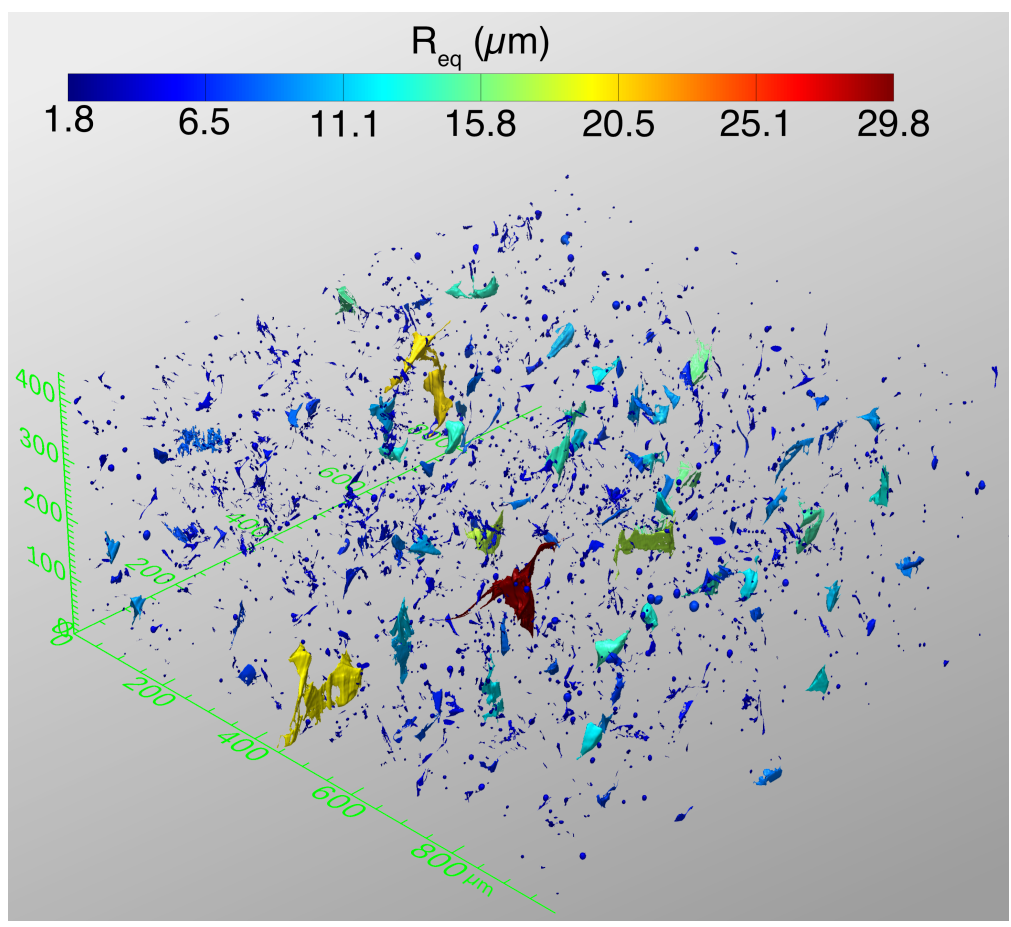

Figure 2. 3D Reconstruction of pores in AM processed 316L. Color represents the spherical equivalent radius of the pore. 